\title{
Wirtschaftsethik und Dritter Sektor
}

\author{
KARL GABRIEL ${ }^{*}$
}

\section{Economic Ethics and the Third Sector}

This article starts with a clarification of the origins and the specificities of the Third Sector and its organizations. In line with actual research interests it analyzes organizations of the Third Sector as actors of the civil society. In the second part it addresses two leading approaches that were developed in Germany as comprehensive conceptions of economic and business ethics. The question is: What can the approaches of Karl Homann on the one side and of Peter Ulrich on the other side contribute to an ethical reflection on the Third Sector and its organizations?

Keywords: Dritter Sektor, Zivilgesellschaft, Ökonomische Theorie der Ethik (Homann), Integrative Wirtschaftsethik (Ulrich)

\section{Einleitung}

Die Forschung zum Dritten Sektor zeichnet sich durch eine hohe Multidisziplinarität aus (vgl. Zimmer 2007: 186). Seit Amitai Etzioni (1973) vom „Third Sector“ sprach, haben viele Soziologen, Politikwissenschaftler und Wirtschaftswissenschaftler sich mit der Thematik eines Raumes bzw. von Organisationen jenseits von Markt und Staat beschäftigt. Die Wirtschaftsethik findet man unter den Disziplinen, die sich durch die Welt jenseits staatlicher Bürokratie und gewinnorientierten Unternehmen herausgefordert fühlten, so gut wie nicht vertreten. Einerseits gibt es in den Sozialwissenschaften eine gewisse Zurückhaltung und Skepsis gegenüber der Wirtschaftsethik. So hatte Niklas Luhmann auch nur Ironie und Spott für das Projekt einer Wirtschaftsethik übrig:

„Ich muß es gleich am Anfang sagen:“ - so eröffnete er ein Referat zur Wirtschaftsethik in Münster - „es ist mir nicht gelungen herauszubekommen, worüber ich eigentlich reden soll. Die Sache hat einen Namen: Wirtschaftsethik. Und ein Geheimnis, nämlich ihre Regeln. Aber meine Vermutung ist, dass sie zu der Sorte von Erscheinungen gehört wie auch die Staatsräson oder die englische Küche, die in der Form eines Geheimnisses auftreten, weil sie geheimhalten müssen, dass sie gar nicht existieren.“ (Luhmann 1993: 134)

Dem Charakter der Wirtschaftsethik als Geheimlehre widerspricht, dass spätestens seit Beginn der 1990er Jahre Publikationen über Wirtschaftsethik ,im konjunkturellen Aufwind“ (Hengsbach 1993: 9) liegen. Bis in die Gegenwart hinein lässt sich von einem gewissen Boom der Wirtschaftsethik sprechen, der aus der Wirtschaft selbst ge-

Prof. Dr. Dr. Karl Gabriel, Institut für Christliche Sozialwissenschaften, Katholisch-Theologische Fakultät der Universität Münster, Hüfferstr. 27, D-48149 Münster, Tel.: +49-(0)251-8332640, Fax: +49-(0)251-30041, E-Mail: gabrielk@uni-muenster.de, Forschungsschwerpunkte: Sozialethik, Wohlfahrtsverbändeforschung, Religionssoziologie, Religion und Sozialstaatsentwicklung. 
speist wird. Die Wirtschaftsethik zeigt aber eine hohe Konzentration auf die wachsenden Legitimationsprobleme der marktlich organisierten Wirtschaft und gewinnorientierter Unternehmen. So ist es nicht verwunderlich, dass die „hybriden Einrichtungen“ (vgl. Evers/Laville 2004) des Dritten Sektors wirtschafts- und unternehmensethisch unterbelichtet geblieben sind.

Der folgende Beitrag setzt bei einer Klärung von Herkunft und Spezifika des Dritten Sektors und seiner Organisationen ein. Dem neueren Forschungsinteresse folgend geht er auf Dritte-Sektor-Organisationen als zivilgesellschaftliche Akteure ein. Im zweiten Teil wendet sich der Beitrag den zwei führenden Ansätzen zu, die im deutschsprachigen Raum umfassende Konzeptionen von Wirtschafts- und Unternehmensethik entwickelt haben: Welchen Beitrag können die Ansätze von Karl Homann einerseits und Peter Ulrich andererseits - so wird gefragt - zu einer ethischen Reflexion des Dritten Sektors und seiner Organisationen leisten?

\section{Dritter Sektor}

Im Begriff Dritter Sektor kommt ein breiter Komplex von Entwicklungen zur Sprache, die auf erschwerte Handlungsbedingungen und neue Grenzen politischer Macht und staatlicher Administration hinweisen (vgl. Gabriel 2001; Birkhölzer et al. 2005; Zimmer/Priller 2005; Zimmer 2007). Die groben Etikettierungen des Wandels als Individualisierung auf der einen und Globalisierung auf der anderen Seite zeigen lediglich die Stoßrichtungen an, aus denen heute die Herausforderungen und Bewährungsproben für den territorial begrenzten Massenstaat kommen. Auf der Rückseite der vielfältigen Prozesse schmerzlicher Fremd- wie auch Selbstbegrenzung des Staates schiebt sich ein sozialer Raum bzw. Sektor in den Vordergrund, der aus der ihm zugedachten Rolle als passives und strukturloses Objekt staatlichen Handelns heraus drängt und auf einen wie immer gearteten Subjektstatus rekurriert.

Als problemanzeigender Begriff verweist die Rede vom Dritten Sektor nicht nur auf Phänomene der Entzauberung des modernen Staates, sondern hat gewissermaßen auch eine zweite Erfahrung von Enttäuschung und Ernüchterung zum Bezugspunkt. Die vom Ende des Staatssozialismus her nahe liegende Erwartung, befreie man die Gesellschaft nur von den Fesseln eines hypertroph gewordenen Staates und überlasse man den Bedarf an Koordination und Kooperation allein dem freien Wettbewerb und Tausch von Marktanbietern sowie -nachfragern, so werde sich die jeweils bestmögliche Lösung von Problemen der Handlungskoordination und des Interessenausgleichs wie von selbst herstellen, hat sich ebenfalls nicht erfüllt. Die theoretisch wie empirisch radikalisierte und erweiterte Systemintegration über den Markt hat der Frage nach den Kräften der Sozialintegration eine neue Dringlichkeit gegeben. Der von den Finanzmärkten ausgehende Druck auf Wirtschaftsunternehmen, dem Profitmotiv verschärft Relevanz zu geben, hat der Bedeutung von Organisationen jenseits der Profitorientierung Nachdruck verliehen. Der bis in die Gegenwart hinein im gesellschaftlichen Test befindliche Versuch, der Marktsteuerung in den Feldern von Kultur, Bildung, Wissenschaft, sozialen Diensten und des Gesundheitswesens mehr Raum zu geben, hat auch den Grenzen einer Vermarktlichung gesellschaftlichen Lebens wieder schärfere Konturen gegeben. 
Neben der horizontalen Blickverschiebung über die Sphären von Wirtschaft und Politik hinaus steht hinter der Artikulation eines ,Dritten Sektors' auch eine neue Aufmerksamkeit für verschärfte gesellschaftliche Probleme in der vertikalen Dimension. Die Gleise, auf denen sich Gesellschaft und Individuum bewegen, streben wie nie zuvor auseinander. Auf der einen Seite verschärft sich die Eigensinnigkeit der tragenden gesellschaftlichen Institutionen, so dass deren innere Logik offenbar nur noch vom distanzierten Beobachter mittels einer hoch abstrakten Systemtheorie einigermaßen begreifbar und einholbar erscheint. Auf der anderen Seite wachsen die Ansprüche der Individuen, die Welt der Institutionen den eigenen Interessen unmittelbar dienstbar zu machen und sich deren Sinnvorgaben nur dort zu Eigen zu machen, wo eine Verschränkung mit eigenen Sinnbezügen glaubwürdig nachvollziehbar erscheint. Entscheidende Bedeutung für die Möglichkeiten gesellschaftlicher Integration einerseits wie der Sinnhaftigkeit individueller Lebensführung andererseits erhält damit die Sphäre zwischen der Welt der Individuen, ihres persönlich-individuellen und familiären Lebens und der Welt der anonymisierten gesellschaftlichen Großinstitutionen. Intermediäre institutionelle Strukturen mit der Fähigkeit zur Vermittlung und zum Spannungsausgleich zwischen Individuum und Gesellschaft lassen sich damit als Engpass gegenwärtiger gesellschaftlicher Entwicklung identifizieren. Mit der Rede vom ,Dritten Sektor' werden auch eine neue Aufmerksamkeit und die Suche nach einem angemessenen konzeptionellen und begrifflichen Zugang zur Sphäre eines ,Zwischen' in der vertikalen Dimension gesellschaftlichen Lebens angesprochen.

\section{Dritte-Sektor-Organisationen}

Für den Dritten Sektor sind Organisationen charakteristisch, die vier Merkmale gemeinsam haben (vgl. Priller/Zimmer 2004; Zimmer/Priller 2005; Zimmer 2007: 179193). Sie besitzen ein Mindestmaß an formeller Struktur, was sie von rein informellen Aktionsformen wie Stammtischrunden etc. unterscheidet. Die Rechtsform als eingetragener Verein, private Stiftung, gemeinnützige GmbH oder gemeinnützige Genossenschaft - so in der Rechtsstruktur Deutschlands - ist das entscheidende Moment. Ihre Zugehörigkeit zum Dritten Sektor impliziert, dass sie eine gewisse Unabhängigkeit und Eigenständigkeit gegenüber dem Staat besitzen. Von Wirtschaftsunternehmen unterscheiden sie sich durch ihre Nichtgewinnorientierung, die zwar die Erzielung von Gewinnen prinzipiell zulässt, nicht aber ihre Ausschüttung an Eigner oder Mitglieder. Gewinne müssen der Reinvestition zugeführt werden. Als viertes konstitutives Merkmal gilt, dass die Mitgliedschaft keine Zwangsmitgliedschaft darstellt, sondern freiwillig zustande kommt. Darüber hinaus können Dritte-Sektor-Organisationen bei ihrer Aufgabenerfüllung auch auf freiwillige Beiträge bei Geldmitteln oder Arbeitsleistung zurückgreifen.

Die Organisationen des Dritten Sektors lassen sich einem dritten Typus von Organisationen zurechnen, die weder öffentliche Ämter noch gewerbliche Unternehmungen darstellen (vgl. Pankoke 2000; Ebertz 2001). Ihre Identität ist gebunden an einen eigenen Stil des Handelns mit eigener Motivation und Rationalität, der sich sowohl vom staatlich-hoheitlichen Machthandeln als auch vom Nutzenkalkül kommerziellen Handelns unterscheidet. Sie agieren als sogenannte Non-Profit-Organisationen mit besonderer Gemeinwohlverpflichtung im Dritten Sektor - neben dem Staat auf der einen 
Seite und dem privatwirtschaftlich organisiertem Markt auf der anderen Seite. Gleichzeitig bilden sie eine Brücke zu den auf wechselseitiger Hilfe beruhenden privatfamiliären Gemeinschaften und deren Logik solidarischen Handelns. In Deutschland lässt sich zum Beispiel an der Entwicklung der Wohlfahrtsverbände in den letzten Jahrzehnten der Bedeutungszuwachs des Dritten Sektors ablesen. In ihrer spezifischen Organisationsform stehen Akteure des Dritten Sektors vor der Aufgabe, verschiedene Handlungslogiken, Motive und Rationalitäten so miteinander zu verbinden, dass sie eine produktive Einheit bilden. Die Bedeutung der Organisationen des Dritten Sektors lässt sich mit Problemen eines Staats- und Marktversagens einerseits, wie auch mit den engen Funktions- und Leistungsgrenzen informeller Hilfeformen andererseits begründen.

Die Organisationen des Dritten Sektors haben es mit einer auffälligen Multifunktionalität zu tun (vgl. Zimmer/Priller 2005: 54-56). Auf der einen Seite erbringen sie Dienstleistungen, insbesondere im sozialen Bereich. Als Dienstleistungserbringer reichen die Dritte-Sektor-Organisationen in den Markt hinein. Gleichzeitig haben sie in der Regel Funktionen einer Interessenvertretung. Als solche organisieren und bündeln sie Interessen und agieren als politische Akteure. In vertikaler Perspektive leisten die Dritte-Sektor-Organisationen in ihrem lokalen, auf den informell-privaten Bereich gerichteten Bezug einen wichtigen Beitrag zur sozial-kulturellen Integration. Dienstleister, Lobbyisten und Sozialintegratoren zugleich zu sein, bedingt für die Dritte-Sektor-Organisationen und deren Management spezifische Probleme der Balance. Stets befinden sie sich in der Gefahr, ihre balancierte Identität nach einer Seite hin aufs Spiel zu setzen.

Dies lässt sich eindringlich am Beispiel der kirchlichen Wohlfahrtsverbände demonstrieren (vgl. Gabriel 2007). Zur Stellung der kirchlichen Wohlfahrtsverbände gehört eine Vielfalt von Aufgaben- und Funktionsbezügen und Vermittlungsleistungen in einem Spannungsfeld, das sich nicht ohne weiteres nach einer Seite hin auflösen lässt, wollen die Verbände nicht ihren spezifischen Ort, ihre Domäne, ihre historisch gewachsene Identität aufs Spiel setzen. Dies gilt gegenüber dem Druck zur Vermarktlichung von Diakonie und Caritas als Dienstleistungsakteure auf einem mehr oder weniger regulierten Markt wie auch gegenüber dem Druck, sich den Interessen staatlichhoheitlichen Handelns zu beugen. Auch der Rückzug auf die Ebene einer charismatisch-informellen Hilfe von Mensch zu Mensch allein würde ihrer Tradition nicht entsprechen. Als kirchliche Akteure kommt für Diakonie und Caritas noch die Aufgabe hinzu, trotz ihrer engen kirchlichen Bezüge, sich auch von den kirchenamtlichen Strukturen nicht ununterscheidbar zu machen. Ein Nachgeben gegenüber dem Verkirchlichungsdruck müsste nicht nur die Handlungsfähigkeit der Verbände in ihrer Funktion als Anwalt und Dienstleister beeinträchtigen, sondern auch ihre Inkulturationsleistungen in die Gesellschaft hinein erschweren.

Steuerungstheoretisch spielt innerhalb der Dritte-Sektor-Organisationen ein dritter Typus von Steuerung neben Markt und Staat eine Rolle, der sich als solidarische Steuerung bezeichnen lässt (vgl. Geller/Gabriel 2004: 17-20). Im idealtypischen Sinne operieren Markt und Staat/Hierarchie unter der Prämisse, dass die beteiligten Akteure primär ihre eigenen Interessen verfolgen und nur durch systemspezifisch generierte Informationen und Sanktionen zu einem Verhalten gebracht werden, das auch Dritten 
nützt. Unter den Bedingungen der Solidarität dagegen gilt gerade diese primär auf eigene Interessen bezogene Disposition als aufgehoben, das Verhalten orientiert sich spontan an angenommenen gemeinsamen Interessen, Normen und Wertorientierungen der sich solidarisch Fühlenden; dementsprechend stellt sich das für Staat wie Markt prekäre Problem der Vermittlung von Zielgrößen und individuellen Bedürfnissen hier nur sehr wenig ausgeprägt (vgl. Kaufmann 2002: 173). Solidarität mildert eine mögliche Differenz zwischen gemeinschaftlichen Zielgrößen und individuellen Interessen ab. Sie gelingt vor allem dann, wenn Selbstinteressen und Gruppeninteressen zusammenfallen. Die Verfolgung individueller Interessen nützt dann gleichzeitig der Gruppe, die Vertretung von Gruppeninteressen stützt die eigenen.

Wichtige Voraussetzungen für die Steuerung und Koordination von Handlungen durch Solidarität sind ein normativer Konsens der Beteiligten und eine gemeinsame Situationsdefinition. Das Informationsproblem solidarischer Verhaltenskoordination wird durch spontane Kommunikation gelöst. In diesen Kommunikationen werden Probleme definiert und Ursachen zugeschrieben. Solidarische Steuerung ist dann besonders erfolgreich, wenn die Beteiligten ein Problem so zuschreiben, dass es nicht als durch Handeln der Beteiligten verursacht erscheint, wenn sie das Problem als auferlegt erleben, es also als außen verursacht betrachten, so dass sie vor einem gemeinsamen Gegenüber stehen. Solidarische Kommunikation ist Kommunikation in direkten Interaktionen. Sie ist an Interpersonalität gebunden und setzt als solche Ich-DuBeziehungen voraus, d. h. ein Ernstnehmen des Anderen in seiner Eigenart als Person, was wiederum voraussetzt, dass man sich gegenseitig kennt. Von hier aus bestimmen sich dann auch die Kriterien, nach denen Situationen und Vorgaben zur Lösung von Problemen ausgelegt werden sollen. Während Gesetze ,sine ira et studio', unter Absehung von der Person, ausgelegt werden und der Markt auf Anonymität beruht, ist für die Anwendung des Steuerungsmediums Solidarität gerade das Ansehen der Person von entscheidender Bedeutung. Von besonderer Bedeutung ist in diesem Zusammenhang das in den Anderen gesetzte Vertrauen. Vertrauen wird demjenigen entgegengebracht, der zuverlässig wirkt. Der Vertrauende macht dem Anderen ein Angebot einer gemeinsamen Zukunft. Doch ist Vertrauen riskant und wird nicht blind gewährt, wenn auch die Vertrauensperson einen Kredit genießt, in dessen Rahmen auch Enttäuschungen ertragen werden. Dieser Rahmen ist aber nicht beliebig groß, sondern er legt Schwellen fest, jenseits derer Vertrauen entzogen wird (vgl. Luhmann 1968).

\section{Dritte-Sektor-Organisationen als zivilgesellschaftliche Akteure}

In der Forschung zum Dritten Sektor spielt gegenwärtig deren Bedeutung für die infrastrukturellen Grundlagen der Zivilgesellschaft eine wachsende Rolle (vgl. Zimmer 2007: 191). Deshalb erscheint gerade in normativer Perspektive der Zusammenhang zwischen Dritte-Sektor-Organisationen und Zivilgesellschaft von Interesse. Gemeinsam sind den unterschiedlichen Konzeptionen von Zivilgesellschaft vier Elemente. Über sie gibt es zumindest die wenigsten Kontroversen (vgl. Kaelble 2004: 270):

(1) Die Zivilgesellschaft ist strikt vom Staat und der zentralen politischen Macht zu unterscheiden. Sie besitzt eigene Institutionen, Organisationen, Netzwerke und Projekte. Darüber hinaus sind spezifische Werte für die Zivilgesellschaft charakteristisch: Zivilität, Gemeinwohlorientierung, Altruismus, Gewaltlosigkeit, 
wechselseitige gleichrangige Anerkennung, Vertrauen und Zivilcourage. Außer vom Staat grenzt sich die Zivilgesellschaft auch vom Markt und von der Privatsphäre ab.

(2) Zu den zentralen Charakteristika der Zivilgesellschaft gehört ihre Autonomie. Sie kann ihre Funktionen nur in Unabhängigkeit von staatlichen Verwaltungen und privaten Interessenorganisationen erfüllen. Der Anspruch der Autonomie gilt auch gegenüber kirchlichen Entscheidungsträgern. Die Zivilgesellschaft ist sowohl in ihren Werten wie auch in ihrem Zugang zu Politik und Öffentlichkeit autonom.

(3) Zivilgesellschaften sind plural strukturiert. Sie bestehen aus vielen Organisationen, Bewegungen, Netzwerken und Projekten. Sie gehorchen nicht einer zentralen Instanz und haben keine zentrale Organisationsspitze. Die Pluralität und Offenheit der Zivilgesellschaft bedingt aber auch ihre spezifische Gefährdung: sie kann ausgenutzt und für administrative Zwecke missbraucht werden. Diktatoren werden versucht sein, sich eine Zivilgesellschaft für eigene Zwecke zu schaffen.

(4) Zivilgesellschaften sind immer mit Öffentlichkeit verbunden. Hartmut Kaelble (2004: 270) spricht davon, dass ohne Verbindung zur Öffentlichkeit eine Zivilgesellschaft nur Gesellschaft wäre. Der Bezug zur Öffentlichkeit ist entscheidend für den spezifischen Einfluss der Zivilgesellschaft auf die Politik. Ohne die politische Öffentlichkeit kann es deshalb auch keine Zivilgesellschaft geben. Der Öffentlichkeitsbezug gilt für lokale Versammlungsöffentlichkeiten bis hin zur Öffentlichkeit auf nationaler und transnationaler Ebene.

Zivilgesellschaften haben - so könnte man zusammenfassen - zwei Brennpunkte (vgl. Adloff 2005: 7-16): Zum einen das Netzwerk von Institutionen, Organisationen, Bewegungen, Initiativen und Einzelakteuren, die nicht primär an politischer Macht oder wirtschaftlichem Gewinn, aber auch nicht ausschließlich an Familien- und Freundschaftsbeziehungen orientiert sind. Zu Akteuren der Zivilgesellschaft werden sie vornehmlich durch ihre Verpflichtung auf Ziele des allgemeinen Interesses und der Unterstützung der Machtlosen und Gefährdeten in der Gesellschaft. Den zweiten Brennpunkt bilden die Werte der Zivilgesellschaft: Zivilität, Zuwendung zum anderen ohne Macht- und Profitinteressen, Prosozialität und caritative Orientierung. Hat man die Zivilgesellschaft vornehmlich als eigenen sozialen Raum oder Sektor im Blick, dann gehören wirtschaftliche und staatliche Organisationen nicht zur Zivilgesellschaft. Orientiert man sich stärker an Zivilität als Wert, kann es zivilgesellschaftliche Elemente auch in Wirtschaftsunternehmen und staatlichen Organisationen geben.

Drei Funktionen und Leistungen der Zivilgesellschaft lassen sich hervorheben (vgl. von Soosten 2001: 1847):

(1) Sie stellt das Feld öffentlicher Einmischung der Bürger und ihrer Selbstorganisation dar. Ihr Raum dient der Mitsprache und Meinungsbildung in öffentlichen Angelegenheiten.

(2) Man erhofft sich von ihr ein kritisches Korrektiv und eine besondere Wachsamkeit gegenüber den Machtkonzentrationen und Verselbständigungstendenzen innerhalb von Wirtschaft und Staat. 
(3) Zu ihren Charakteristika gehört es, eine besondere Sensibilität für die Belange der Schwachen und Benachteiligten in der Gesellschaft auszubilden. Gerade darin kommt ihre Integrationsleistung zum Ausdruck.

In der Regel bleibt der Staat ein vorausgesetzter Bezugspunkt und eine Stütze der Zivilgesellschaft. Sie soll - außer in einigen radikal-demokratischen Konzepten - die formellen politischen Institutionen nicht ersetzen. Sie soll kritisches Korrektiv, aber auch ziviler Treibstoff des Staates sein. Die Nähe zur Idee der Subsidiarität ist hier unverkennbar.

\section{Dritte-Sektor-Organisationen und die Wirtschafts- und Unternehmens- ethik Karl Homanns}

Im deutschsprachigen Raum sind es vornehmlich zwei Ansätze, die dem wirtschaftsund unternehmensethischen Diskurs ihr Gepräge geben: der welfaristische Ansatz von Karl Homann und der diskursethische von Peter Ulrich (vgl. Gabriel 2007b: 160-182). Es handelt sich um zwei komplexe, differenziert ausgearbeitete Entwürfe, die die wirtschafts- und unternehmensethische Perspektive eng miteinander verknüpfen. Sie sollen im Folgenden auf ihren jeweiligen Beitrag für das Feld der ethischen Reflexion des Dritten Sektors und seiner Organisationen geprüft werden.

Homann betont, dass die Wirtschaftsethik von der Einsicht in die Gesellschaftsstrukturen moderner Gesellschaften ausgehen müsse (vgl. Homann/Blome-Drees 1992; Homann 1993; Homann 1997; Homann 2002a). Während die philosophische Ethik entweder von vormodernen Vorstellungen von Gesellschaft ausgehe oder sich auf die Diagnose Pluralismus und die Therapie Integration über die Begründung von Normen beschränke, will Homann für seinen Ansatz, der Wirtschaftsethik als Ökonomik, von modernen Gesellschaftsstrukturen ausgehen und ihnen in der Ethik punktgenau Rechnung tragen. Als Grundstrukturen moderner Gesellschaften betrachtet er die funktionale Differenzierung der Gesellschaft, die Individualisierung, die Ausbildung von Organisationen und die exorbitante Zunahme von Mobilität (vgl. Homann/Blome-Drees 1992: 1-13).

Schon Adam Smith - so Homann - habe mit seinem Hauptwerk „The Wealth of Nations“ auf die Entwicklung hin zu modernen Gesellschaften reagiert. Unter den strukturellen Bedingungen moderner Gesellschaften wird für Homann die Implementierung moralischer Normen zum entscheidenden Problem. Diese könne nur auf das unmittelbar handlungsleitende Motiv individuellen Vorteilsstrebens gegründet werden. „Normen werden befolgt“, so Homann, ,und nur dann befolgt, wenn ihre Befolgung dem jeweiligen Akteur größere individuelle Vorteile verheißt als ihre NichtBefolgung" (Homann 2002a: 26). Zur Moderne gehört, dass die Menschen auch ihre Handlungsbedingungen in die Hand bekommen. Deshalb muss für Homann eine moderne Ethik zweistufig angelegt sein, nämlich als Handlungsethik und als Bedingungsethik. Insofern in den Spielregeln, in der Rahmenordnung, die Optionen und Restriktionen des Handelns geschaffen werden, hat für Homann die Ethik der Handlungsbedingungen den Vorrang. Das Handeln selbst folgt dann den Anreizen, also der ökonomischen Logik. Damit wird erkennbar, dass sich für Homann die zentralen Fragen der Moral von der Handlungs- auf die Systemebene, von den Spielzügen in die Spielregeln verlagern. 
„Der systematische Ort der Moral“ - so Homanns zentrale These - „in einer Markwirtschaft ist die Rahmenordnung.“ (Homann/Blome-Drees 1992: 35)

Handlungs- wie Ordnungsethik müssen für Homann konsistent auf Vorteilskalkulationen aufbauen. Zwei Gründe sind dafür maßgebend: Unter modernen gesellschaftlichen Bedingungen wird für Homann das ältere, an überschaubare Verhältnisse gebundene System der sozialen Kontrolle ersetzt durch ein System, das auf der „Selbstkontrolle entlang den eigenen Interessen, entlang den Anreizen“ (Homann 2002a: 30) beruht. Mit der Selbstkontrolle entlang der Kalkulation eigener Vorteile besitzen moderne, anonyme Großgesellschaften das notwendige funktionale Äquivalent, den notwendigen Ersatz für die lückenlose soziale Kontrolle von Angesicht zu Angesicht in einfachen Gesellschaften. Er fährt fort:

„Weit entfernt davon, das Streben nach eigenen Vorteilen als sozial schädlich zu denunzieren, stellt die lückenlose Selbstkontrolle entlang der eigenen Interessen das einzige durchgängig wirksame Instrument der sozialen Kontrolle in modernen Gesellschaften dar. Moral folgt den ,Anreizen', wird ,Anreizmoral'.“ (ebd.: 30)

Die Kontrolle durch internalisierte Moral muss für Homann scheitern, weil moralische Vorleistungen im Wettbewerb von Marktwirtschaften ausbeutbar sind. Deshalb avanciert die Verfolgung von Eigeninteressen zu der auf moderne Gesellschaften präzise zugeschnittenen Form der sozialen Kontrolle.

Moderne Gesellschaften - so Homann - sind keine stationären Gesellschaften, die gewissermaßen auf einem Nullsummenspiel beruhen, sondern Wachstumsgesellschaften, die auf einem Positivsummenspiel beruhen. Moderne Marktwirtschaften haben für Homann auf der Grundlage einer Verfolgung der eigenen Interessen jedes Einzelnen unter einer klug geschnittenen Rahmenordnung mit forciertem Wettbewerb einen bisher nicht erreichten Wohlstand geschaffen.

„Marktwirtschaft mit Wettbewerb“ - so Homann in provokativen Formulierungen

-,,löst die alte Caritas, die alte Mildtätigkeit, ab, sie wird zur effizientesten Form

der Caritas, die die Weltgeschichte bisher gesehen hat.“ (Homann 2002b: 16)

Die Ökonomik arbeitet in ihrer Methodik mit einem sehr weiten Vorteilsbegriff. Er kann Einkommen, Vermögen, Besitz, aber auch Muße, gutes Leben’ in Gemeinschaft mit anderen umfassen. Ist mit dem Vorteilsdenken der Schlüssel für die individuelle Handlungssteuerung als Ersatz für die nicht mehr funktionierende Motivmoral gefunden, so müssen für Homann die institutionellen Regelsysteme so gebaut bzw. umgebaut werden, dass sie über das Vorteilsdenken der einzelnen zu gewünschten Ergebnissen führen.

Die Unternehmensethik Homanns bleibt eng auf die aufgezeigten wirtschaftsethischen Prämissen bezogen. Da der systematische Ort der Moral für Homann in der Rahmenordnung liegt, bedarf es eigentlich keiner Unternehmensethik. Sie kommt erst ins Spiel, wenn man in den Blick nimmt, dass „die Voraussetzung einer vollkommenen Rahmenordnung (...) aus pragmatischen und systematischen Gründen niemals vollständig gegeben“ (Homann/Blome-Drees 1992: 114) ist. Die Rahmenordnung komme stets in Zeitverzug, habe immer unter Kontrollproblemen zu leiden und zudem müsse mit einem wachsenden Verfall der ordnungspolitischen Kompetenz der Politik gerechnet werden. Erst wo die ordnungspolitischen Defizite auf die Hand- 
lungsebene durchschlagen, fällt die im Normalfall an die Ordnungsebene abgegebene moralische Verantwortung an die Unternehmen zurück. Auch die Unternehmensethik ist für Homann deshalb primär als Institutionenethik und nicht als Individualethik bzw. als Handlungsethik des einzelnen Managers/Unternehmers zu konzipieren. „Unter den Bedingungen der modernen (Markt-) Wirtschaft lässt sich eine Unternehmensethik sinnvoll nur aus ordnungsethischer Perspektive entwickeln“ (ebd.: 121). Für die Unternehmensebene zieht Homann die Konsequenz, systematisch zwischen zwei Typen von Spielzügen zu unterscheiden: zum einen den Handlungstyp des gewinnorientierten unternehmerischen Handelns im Wettbewerb. Dieses ist von moralischen Ansprüchen durch die Rahmenordnung entlastet. Davon unterscheidet Homann die politischen Spielzüge der Unternehmen, die darauf gerichtet sind, die Rahmenordnung im Interesse aller Unternehmen zu verbessern. Homann betont, dass „neben allen Unterschieden sowohl der originär ökonomische als auch der politische Handlungstyp interessengeleitet ist" (ebd.: 123).

Was können die Akteure und Organisationen des Dritten Sektors aus der Wirtschaftsund Unternehmensethik Karl Homanns lernen? Die Individualethik - dies gilt auch für die Reflexion der Handlungsbedingungen im Dritten Sektors - bedarf des Bezugs zu einer Institutionenethik. Organisationen des Dritten Sektors haben die Neigung, ihre individuellen Akteure moralisch zu überlasten. Homanns Perspektive, die Institutionenebene aus der ethischen Reflexion nicht auszublenden, sondern ihr für die Ethik einen zentralen Stellenwert einzuräumen, besitzt deshalb gerade für die Dritte-SektorOrganisationen eine hohe Plausibilität. Gerade in dem Maße, in dem Dritte-SektorOrganisationen als Dienstleister in den Markt hineinreichen, sind sie darauf angewiesen, dass der Ordnungsrahmen stimmt, in dem sie agieren und zur wettbewerblichen Orientierung angehalten werden. So haben die politisch hoch regulierten Sozialmärkte innerhalb der ambulanten Pflege zum Beispiel ein Defizit an Moral, das nur mit groBer Mühe und Tendenzen zur Selbstausbeutung durch die Akteure vor Ort kompensiert werden kann (vgl. Geller/Gabriel 2004: 293-303). Organisationen des Dritten Sektors müssen deshalb den politischen Handlungstyp im Sinne Homanns reflektiert entwickeln und gezielt zum Einsatz bringen.

Ein zweiter Gedanke aus der Wirtschafts- und Unternehmensethik Homanns, der für Dritte-Sektor-Organisationen ein Anregungs- und Reflexionspotential enthält, ist die Bedeutung der Implementation für die Ethik. Ethische Maximen bedürfen der Implementation in konkreten Kontexten, um wirksam werden zu können. Es dürfte auch sinnvoll sein, bei der Implementationsproblematik die Dimension der Anreize nicht aus den Augen zu verlieren. Auch in Dritte-Sektor-Organisationen dürfen institutionelle Arrangements und moralische Anforderungen an die Individuen nicht in Widerspruch zueinander stehen und sich gegenseitig dementieren. Für die Implementation entscheidend ist die Frage, ob sie sich wechselseitig unterstützen, was eine kluge Anreizpolitik voraussetzt.

Aus ethischer Perspektive lassen sich grundsätzliche Einwände gegen die Wirtschaftsund Unternehmensethik Homanns formulieren (vgl. Gabriel 2007b: 106). Vorstellungen einer guten Gesellschafts- und Wirtschaftsordnung reduzieren sich bei Homann faktisch auf eine Option für den Status quo. Als Maßstab gilt das subjektiv bewertete Nutzenniveau des Einzelnen. Handlungen werden ausschließlich nach ihren Folgen 
für das Wohlbefinden des Einzelnen bewertet. Differenzierungstheoretisch muss es befremden, dass der Imperialismus der homannschen ökonomischen Handlungstheorie die Handlungsbedingungen des Wirtschaftssystems auf alle Handlungsbereiche überträgt. Homann findet damit keinen Zugang zur Selbstzwecklichkeit des Menschen und der darin begründeten Freiheits- und Beteiligungsrechte, deren Beachtung ein Vorrang vor allen Wohlfahrtssteigerungen zukommt. „Der moral point of view wird so zweifellos verfehlt" (Ulrich 2001: 115; vgl. Goertz 2004: 213f.).

Mit Bezug auf den Dritten Sektor ist anzumerken, dass Homanns Wirtschaftsethik die Spezifika des Dritten Sektors in Differenz zu Markt und Staat nicht angemessen zu reflektieren vermag. In seiner Ordnungstheorie spielt eine Sphäre jenseits von Markt und Staat keine Rolle. Dritte-Sektor-Organisationen müssen ihm als vormoderne Phänomene erscheinen, die in die Moderne hineinragen. Den Anforderungen, die an alle Organisationen in globalisierten und individualisierten Gesellschaften gestellt werden, können Dritte-Sektor-Organisationen für Homann nicht gerecht werden. Ein systematischer Ort in der Gesellschaft ist für sie nicht vorgesehen. Das politische Handeln von Unternehmen bleibt auch dort, wo es um die politische Rahmenordnung geht, strikt an den Interessen des Unternehmens orientiert. Eine Gemeinwohlorientierung, die diesen Namen verdient, lässt der Ansatz Homanns weder auf der Ebene des unmittelbaren Veränderungshandelns noch auf der Ebene des politischen Handelns zu. Dürfte diese Perspektive schon für reine Wirtschaftsunternehmen zu problematischen Konsequenzen führen, so erweist sie sich insbesondere für Organisationen als unangemessen, zu deren Identität ein Gemeinwohlbezug gehört. Besonders problematisch erscheint, dass Homanns Ansatz für eine Pluralität von Handlungslogiken, die zum Ausgleich gebracht werden müssen, keine Sensibilität besitzt. Die universal lauernden Dilemmastrukturen lassen für Homann nur eine einzige Handlungslogik zu. Insofern sind die Folgen, die ein breites Eindringen der wirtschafts- und unternehmensethischen Perspektive Homanns in das handlungsleitende Wissen der Führungskräfte von Dritte-Sektor-Organisationen besäße, als problematisch einzuschätzen.

\section{Der Dritte Sektor und die Wirtschaftsethik Peter Ulrichs}

Die integrative Wirtschaftethik Peter Ulrichs ist der zweite Ansatz, auf den ich mich beziehen möchte (vgl. Ulrich 2001; Ulrich 2000: 553-567). Ulrich will dem diskursethisch begründeten Moralprinzip im Handlungsbereich der Wirtschaft zur Geltung verhelfen. Er zielt - auf eine Kurzformel gebracht - an, die ,ökonomische Ratio ,zur (praktischen) Vernunft" [zu] bringen“ (Ulrich 2000: 555). Wo davon ausgegangen wird, dass die ökonomische Vernunft - der nutzenmaximierende Einsatz knapper Mittel - schon die ganze Vernunft ausmacht, erhält die Ökonomik die Funktion einer Weltanschauung, wird zum Ökonomismus. Den Ökonomismus hält Ulrich für die Großideologie der Gegenwart. Entsprechend versteht er die Ökonomismuskritik als die „systematisch erste und vordringliche Aufgabe“ (ebd.: 556) seiner Wirtschaftsethik.

Die Wirtschaftsethik umfasst für Ulrich vier systematische Grundaufgaben (vgl. Ulrich 2001: 16; Ulrich 2000: 556): 
(1) Entwicklung eines Ansatzes einer Vernunftethik des Wirtschaftens aus einer tragfähigen philosophischen Vernunftethik.

(2) Kritik der reinen ökonomischen Vernunft und ihrer normativen Überhöhung zum ,Ökonomismus'.

(3) Beschreibung der normativen Grundorientierungen eines lebensdienlichen und das heißt eines sinnvollen und legitimen Wirtschaftens.

(4) Konzeptionelle Verortung der Moral des Wirtschaftens in einer modernen Gesellschaft (wirtschaftsethische Topologie).

Ulrich bezieht sich auf die spezifisch humane Moralität des Menschen. In der Tradition der philosophischen Anthropologie spricht er vom Menschen als das ,sich selbst feststellende Wesen" (Ulrich 2000: 556), das nach Gründen zu entscheiden vermag. Moralische Gründe kommen immer dann ins Spiel, wenn es um die grundlegenden, normativen Leitideen und Bedingungen des Menschseins, um seinen Status als selbstbestimmtes Subjekt und die wechselseitige Achtung und Anerkennung der Individuen in diesem Anspruch geht.

Den moralischen Standpunkt einzunehmen heißt dann für Ulrich, dass die gegenseitige Anerkennung der Menschen als Wesen gleicher Würde für das eigene Handeln als verbindliches Kriterium gilt und dass die gegenseitige Anerkennung auch das entscheidende Kriterium für die Gestaltung der Regeln des Zusammenlebens bildet. Chance und Aufgabe einer humanistischen Vernunftethik sieht Ulrich darin, die universale normative Logik der Zwischenmenschlichkeit zu explizieren. Sie wurzelt in einem kognitiven Moment als Grundlage aller Moralität: nämlich in der Fähigkeit des Menschen zum gedanklichen Rollentausch. Im gedanklichen Rollentausch, also dass ich mich aus den Augen der Anderen zu sehen vermag und umgekehrt, wird die kritische Reflexion des eigenen und fremden Handelns möglich. Wenn ich - so die Argumentation Ulrichs - dem anderen das moralische Recht auf Achtung der Voraussetzungen seiner Würde und seines Subjektseins bestreite, so widerrufe ich auch meinen eigenen Anspruch.

Die Suche nach einem Verfahren zur unparteilichen Begründung zwischenmenschlicher Verbindlichkeiten von Rechten und Pflichten prägt die Vernunftethik seit ihrem Beginn - bis zur heutigen Diskursethik. In der Diskursethik erhält der Vernunftstandpunkt der Moral die Form einer verständigungsorientierten Einstellung, die einen Vorrang vor der Orientierung am strategischen Erfolg besitzt, und die Ausprägung eines öffentlichen Diskurses als dem ideellen Ort der Moral in der modernen Gesellschaft. Für Ulrich dürfen Diskursethik und das diskursethische Verfahren aber nicht mit einer Konsensethik verwechselt werden (vgl. Ulrich 2001: 78-94).

Auf eine Vernunftethik des Wirtschaftens bezogen heißt das für Ulrich: Es geht einer solchen Wirtschaftsethik um „die Legitimitätsbedingungen wirtschaftlichen Nutzens-, Vorteils- oder Erfolgstrebens im Lichte der moralischen Rechte (d. h. berechtigte Ansprüche) aller Betroffenen" (Ulrich 2000: 557). Nicht das Erfolgsstreben als solches wird ethisch problematisiert, sondern ob es gegenüber jedermann vertretbar ist. Insofern ist die Orientierung an einem ethisch integrierten Erfolgsstreben gemeint. Für die Lösung von Anspruchskonflikten auf dem Feld der Wirtschaft gelten dann zwei Regeln: 
(1) Das Wirtschaften muss gegenüber den Betroffenen verantwortbar sein;

(2) die Ansprüche der Betroffenen auf Selbstbegrenzung des wirtschaftlichen Akteurs müssen für ihn zumutbar sein angesichts seiner legitimen Ansprüche auf existentielle Selbstbehauptung.

Der Vorrang des moralischen Gesichtspunkts vor dem Aspekt des Erfolgs und der Effizienz des Handelns steht bei Ulrich für den Primat der Ethik als ethische Integration ökonomischer Rationalität. Als ökonomisch vernünftig im Sinne Ulrichs kann damit jede Handlung oder Institution gelten, ,die freie und mündige Personen in der vernunftgeleiteten Verständigung unter allen Betroffenen als legitime Form der Wertschöpfung bestimmt haben könnten“ (ebd.: 558). Diese sozial-ökonomische Rationalitätsidee formuliert für Ulrich den ,moral point of view’ einer Vernunftethik des Wirtschaftens.

Ulrich möchte sich mit diesem Standpunkt abgrenzen von dem in der Ökonomie vorherrschenden ethischen Skeptizismus oder ,Non-Kognitivismus', der davon ausgeht, dass für alle Menschen gültige, universale Gesichtspunkte ethischer Vernunft nicht begründbar sind. In die Reihe der ethischen Skeptizisten rechnet Ulrich auch Karl Homann, der mit seiner normativen Ökonomik bei einer Moralbegründung aus Interessen lande. Mit Blick auf Homann spricht Ulrich von einer funktionalistischen Wirtschaftsethik, da sie dazu neige, die systemischen Funktionsbedingungen der modernen Wirtschaft normativ aufzuladen. Ulrich möchte in seiner Wirtschaftsethik nach den Legitimitätsbedingungen des Wirtschaftssystems selbst fragen, während es Homann lediglich um die Implementierung von Moral unter den fraglos vorausgesetzten Funktionsbedingungen eines idealisierten marktwirtschaftlichen Systems gehe.

Die moderne Wirtschaftsethik erhält bei Ulrich die Aufgabe, den Normenkonflikt zwischen dem vernunftethischen und dem (normativ-)ökonomischen Rationalitätsanspruch zu bearbeiten. In den beiden Rationalitätstypen spiegeln sich für Ulrich gleichzeitig reale geschichtliche Rationalisierungsprozesse wider. Den Ansatz seiner Vernunftethik des Wirtschaftens fasst Ulrich folgendermaßen zusammen:

„Es geht um zukunftsweisendes Orientierungswissen für die Einbindung der institutionell entfesselten und normativ enthemmten, eigensinnig gewordenen ökonomischen Rationalisierungsdynamik in Grundsätze und Leitideen einer lebensdienlichen (d. h. legitimen und sinnvollen) Gestaltung des gesellschaftlichen ,Wirtschaftslebens'.“ (ebd.: 558)

Für Ulrich bedarf es einer kulturellen Reorientierung an einem noch zu entwickelnden ,integrativen' Wirtschaftsstil. Das sinngebende Formalziel des sozio-ökonomischen Fortschritts bestehe in der Indienstnahme hoher Produktivität für die Erweiterung der Vielfalt freier und kultivierter Entfaltungsmöglichkeiten. Dies setzt für Ulrich eine „Kultur des Genug-haben-Könnens, der Selbstbegrenzung des eigenen Nutzen-, Erfolgs- oder Vorteilsstrebens aus Gründen einer kultivierten Lebensform“ (ebd.: 563) voraus. Wie der ,Geist des Kapitalismus’ erst die ökonomische Modernisierung möglich gemacht habe, so bedarf es für Ulrich heute eines ,neuen Geistes', eines neuen motivbildenden Wirtschaftsethos. Der Wandel des Wirtschaftsethos braucht für Ulrich die Unterstützung durch eine „Sachzwangsbegrenzungspolitik“ (ebd.: 563). Nicht überall muss Wettbewerb herrschen und allein der Markterfolg zählen. Es be- 
steht die Möglichkeit, durch einen normativen Rahmen allgemeinverbindlicher Spielregeln Wettbewerb und Markt zu begrenzen: z. B. die Bereiche, in denen Wettbewerb herrschen soll, zu beschränken und die Einzelnen mit subjektiven Rechten auszustatten und damit die Tauschvertragsvoraussetzungen zu verändern. Heute - darüber ist sich Ulrich im Klaren - sei dies erfolgreich nur noch auf supranationaler Ebene zu institutionalisieren. Der mögliche Ausbruch aus der Ökonomie der Lebensnot werde sich nicht einfach als Resultat von Wirtschaftswachstum und Produktivitätsfortschritt einstellen, „sondern erst auf der Basis einer wirtschaftsethisch aufgeklärten Ordnungspolitik, die sich an den Gesichtspunkten eines guten Lebens und gerechten $\mathrm{Zu}$ sammenlebens freier Bürger orientiert" (ebd.: 564).

Für eine wohlgeordnete Gesellschaft freier und gleicher Bürger - so Ulrich im Anschluss an Rawls - ist der Vorrang der Gerechtigkeit im Sinne der gleichen Grundrechte und Grundfreiheiten für alle konstitutiv. Gleichzeitig geht es auch um die sozio-ökonomischen und sozio-kulturellen Voraussetzungen dieser allgemeinen Freiheit. Für Ulrich darf das Rawls'sche Differerenzprinzip nicht auf Gerechtigkeit, im Sinne der Pareto-Effizienz, reduziert werden, weil sonst der jeweilige ,Status quo' voll auf die Ergebnisse durchschlage. Deshalb bedarf der freie Markt der Einbindung in rechtsstaatlich gewährleistete und real lebbare Bürgerrechte als Sicherung des Status als vollwertige Bürger für alle. Die Bürgerrechte - so Ulrich im Anschluss an Dahrendorf - sind unbedingte, an den Bürgerstatus gebundene Anrechte, „die die Kräfte des Marktes zugleich überschreiten und in ihre Schranken verweisen“" (ebd.: 564).

Eine voll entwickelte Bürgergesellschaft besteht für Ulrich noch nicht. In ihr existieren Wirtschaftsbürgerrechte im Sinne sozio-ökonomischer Rechte, die den Bürger vor entwürdigender existenzieller Not und sozialer Benachteiligung schützen und ihm eine angemessene Teilhabe am volkswirtschaftlichen Kapital, Produktionsprozess und -ergebnis sichern. Eine kompensatorische Sozialpolitik könne solche Bürgerrechte nicht ersetzen. Dabei sei die Ausgestaltung der Bürgerrechte im Sinne der Wirtschaftsbürgerrechte Sache der Bürger selbst.

Ulrichs Wirtschaftsethik - so lässt sich zusammenfassen - geht von einer Kritik der normativen Implikationen des wirtschaftlichen Handlungsbereichs aus. Ulrich verschafft sich mit der Aufdeckung der impliziten Normativität der neoklassischen Ökonomik und der Kritik des herrschenden ökonomischen Determinismus und Reduktionismus im Handlungsbereich Wirtschaft die Voraussetzungen für seinen Entwurf lebensdienlichen Wirtschaftens. In Ulrichs Perspektive muss eine Ethik des einzelnen Wirtschaftsbürgers Hand in Hand mit einer Ordnungsethik gehen, die auch eine Begrenzung und Lenkung des Marktes im Interesse der Lebensdienlichkeit vorsieht. Ulrich gibt in seiner integrativen Unternehmensethik der Legitimität und Verantwortbarkeit unternehmerischen Handelns gegenüber allen potentiell Betroffenen den Vorrang vor der Regel der Gewinnmaximierung. Die großen Unternehmen will Ulrich als quasi-öffentliche Institutionen betrachtet wissen, die zu einem Stakeholder-Dialog und zu einer deliberativen Unternehmenspolitik verpflichtet seien.

Schon auf den ersten Blick wird deutlich, dass es für Dritte-Sektor-Organisationen eine Reihe von naheliegenden Anknüpfungspunkte in Bezug auf die integrative Wirtschafts- und Unternehmensethik Peter Ulrichs gibt. Ulrich betrachtet den öffentlichen Diskurs als den ideellen Ort der Moral in modernen Gesellschaften. Dritte-Sektor- 
Organisationen verstehen sich in einem ihrer Funktionsbezüge als organisatorisches Substrat des öffentlichen Diskurses. Die Wirtschaftsethik Ulrichs sieht insofern für die Dritte-Sektor-Organisationen als Akteure zivilgesellschaftlicher Öffentlichkeit einen unverzichtbaren Ort in modernen Gesellschaften vor. Sie gehören zu dem Raum, in dem auf vernunftethischer Basis die Anspruchskonflikte unternehmerischen Handelns ausgetragen werden können. Wo in einem Diskursverfahren um die Verantwortbarkeit und Legitimität der Wertschöpfung vor dem Forum aller Betroffenen gerungen wird und mit der Zumutbarkeit für die Unternehmenseigner zum Ausgleich gebracht werden muss, reicht das Wirtschaftsunternehmen in die Sphäre des zivilgesellschaftlich geprägten Dritten Sektors hinein. Ulrichs scharfe Kritik des Ökonomismus, seine ethische Begründung für die Begrenzung und Unterordnung des Gewinnprinzips unter das Prinzip des gerechten Ausgleichs der Ansprüche aller, legt die Grundlagen für eine Sphäre jenseits des Marktes und seiner Logik. Dazu bedarf es in der Perspektive Ulrichs einer ,Sachzwangbegrenzungspolitik', die einen Primat der politischen Ethik vor der Logik des Marktes voraussetzt. Die Gewährung von Wirtschaftsbürgerrechten, die auch den marktlogischen Ausgrenzungsprozessen gegenüber den am Markt Erfolglosen standzuhalten vermögen, dürfte zu den Voraussetzungen der Entwicklung des Drittens Sektors gehören. Ohne Grenzen der Markvergesellschaftung und der Kommodifizierung der Arbeit bleiben die sozialen Voraussetzungen eines Dritten Sektors prekär. Ulrichs Unternehmensethik ist in der Lage, der ethischen Reflexion und Orientierung von Dritte-Sektor-Organisationen wichtige Impulse zu geben. Das unternehmerische Handeln von Dritte-Sektor-Organisationen bezieht seine Legitimation aus der Verantwortbarkeit gegenüber allen Betroffenen. Dies gilt auch dort, wo die Gewinne nicht an Eigner ausgeschüttet werden, sondern zur Reinvestition vorgesehen werden. Im Sinne Ulrichs muss sich die Unternehmenspolitik am Stakeholder-Dialog unter Einbezug aller Betroffenen orientieren. Eine deliberative Unternehmenspolitik gehört deshalb zu den grundlegenden Maximen einer Unternehmensethik von Dritte-Sektor-Organisationen.

Ulrich erhebt den Anspruch, eine Vernunftethik des Wirtschaftens zu entwickeln (vgl. Gabriel 2007b: 179). In seiner Kritik an den normativen Implikationen des Handlungsbereichs Wirtschaft und im grundlagentheoretischen Rückgriff auf die Diskursethik löst Ulrich diesen Anspruch auch ein. Ethisch betrachtet sind seine Vorstellungen eines lebensdienlichen Wirtschaftens aber eindeutig einer Ethik guten Lebens zuzuordnen und können deshalb nur einen partikularen und keine universalen Geltungsanspruch erheben (vgl. Emunds 2005: 216). Hinsichtlich der Sicht des Handlungsbereichs Wirtschaft hat Ulrich die Tendenz, die Vorteile der Entkoppelung der Koordination wirtschaftlichen Handelns über monetäre Folgen vom kommunikativen Handeln nicht angemessen in den Blick zu bekommen. Es hat den Anschein, als ziele er gewissermaßen eine neue Verkoppelung beider an. So gerät er in Gefahr, unreflektierte ethische Forderungen an die Wirtschaftsakteure zu richten, die nicht als moralische Pflichten zum wirtschaftlichen Handeln, sondern als Pflichten zum politischen Handeln betrachtet werden sollten.

Für das Verhältnis von Ethik und Dritter Sektor bzw. Dritte-Sektor-Organisationen ergibt sich daraus, dass sich die Problematik im Verhältnis zum Ansatz Karl Homanns gewissermaßen umkehrt. Hatte sich oben gezeigt, dass Homann letztendlich das wirt- 
schaftliche und unternehmerische Handeln eindimensional nur als strategisches Interessenhandeln konzipiert, so besitzt Ulrich die Neigung, die Systemlogik wirtschaftlichen Handelns der vernunftethischen Rationalität so stark unterzuordnen, dass der Konflikt zwischen beiden nach der anderen Seite hin aufgelöst erscheint.

Die Perspektive Ulrichs - so lässt sich resümieren - eröffnet für die Sphäre des Dritten Sektors und seiner Organisationen eine breite Grundlage, ihren Ort in der modernen Gesellschaft ethisch zu reflektieren und legitimierbare Handlungsoptionen zu gewinnen. Gleichzeitig sollte sich die ethische Reflexion stärker als dies bei Ulrich erkennbar ist, am gleichberechtigten Ausgleich und an der Vermittlung der differenten Logiken strategischen und kommunikativen Handelns orientieren.

\section{Literaturverzeichnis}

Adloff, F. (2005): Zivilgesellschaft. Theorie und politische Praxis, Frankfurt a. M./New York: Campus.

Birkbölzer, K./ Klein, A./ Priller, E./ Zimmer, A. (2005): Theorie, Funktionswandel und zivilgesellschaftliche Perspektiven des Dritten Sektor/Dritten System - Eine Einleitung, in: Birkhölzer, K./ Klein, A./ Priller, E./ Zimmer, A. (Hrsg.): Dritter Sektor/Drittes System. Theorie, Funktionswandel und zivilgesellschaftliche Perspektiven, Wiesbaden: VSVerlag, 9-15.

Ebertz, M. N. (2001): Wohlfahrtsverbände als intermediäre Solidaritätsschöpfer, in: Gabriel, K. (Hrsg.): Dritter Sektor. Jahrbuch für Christliche Sozialwissenschaften, Bd. 42, Münster: Regensberg, 118-132.

Emunds, B. (2005): Grundlagen einer Ethik internationaler Finanzmärkte, Habilitationsschrift an der Katholisch-Theologischen Fakultät der Universität Münster, Münster.

Etzioni, A. (1973): The Third Sector and Domestic Mission, in: Public Administration Review, 33, 314-323.

Evers, A./ Laville, J.-L. (2004): Social Services by Social Enterprises: On the Possible Contributions of Hybrid Organizations and a Civil Society, in: Evers, A./ Laville, J.-L. (Eds.): The Third Sector in Europe, Cheltenham: Edward Elgar, 237-255.

Gabriel, K. (2001): Vorwort, in: Ders. (Hrsg.): Der Dritte Sektor. Jahrbuch für Christliche Sozialwissenschaften, Bd. 42, Münster: Regensberg, 7-10.

Gabriel, K. (2007a): Caritas und Sozialstaat unter Veränderungsdruck. Analysen und Perspektiven, Hamburg: Lit.

Gabriel, K. (2007b): Zur Relevanz güterethischer Ansätze in der Wirtschaftsethik, in: Reuter, H.-R./ Meireis, T. (Hrsg.): Das Gute und die Güter. Studien zur Güterethik, Berlin: Lit, 160-182.

Geller, H./Gabriel, K. (2004): Ambulante Pflege zwischen Familie, Staat und Markt, Freiburg i. Br.: Lambertus.

Goertz, St. (2004): Weil Ethik praktisch werden will. Philosophisch-theologische Studien zum Theorie-Praxis-Verhältnis, Regensburg: Pustet.

Hengsbach, F. (1993): Wirtschaftsethik. Aufbruch, Konflikte, Perspektiven, 2. Aufl., Freiburg i. Br.: Herder.

Homann, K./ Blome-Drees, F. (1992): Wirtschafts- und Unternehmensethik, Göttingen: Vandenhoek. 
Homann, K. (1993): Wirtschaftsethik. Die Funktion der Moral in der modernen Wirtschaft, in: Wieland, J. (Hrsg.): Wirtschaftsethik und Theorie der Gesellschaft, Frankfurt a. M.: Suhrkamp, 32-53.

Homann, K. (1997): Sinn und Grenze der ökonomischen Methode in der Wirtschaftsethik, in: Aufderheide, D./ Dabrowski, M. (Hrsg.): Wirtschaftsethik und Moralökonomik. Normen, soziale Ordnung und der Beitrag der Ökonomik. Schriften des Vereins für Sozialpolitik, N. F., Berlin: Duncker, 11-42.

Homann, K. (2002a): Ökonomik: Fortsetzung der Ethik mit anderen Mitteln, in: Siebeck, G. (Hrsg.): Artibus ingenuis, Tübingen, wiederabgedruckt in: Folani, A./ Nacke, St. (Hrsg.): Wirtschaftsethik im Diskurs. Eine kritische Auseinandersetzung mit Karl Homann, Münster: Lit., 19-50.

Homann, K. (2002b): Wirtschaft und Moral - eine Neubestimmung ihres Verhältnisses, in: Folani, A./ Nacke, St. (Hrsg.): Wirtschaftsethik im Diskurs. Eine kritische Auseinandersetzung mit Karl Homann, Münster: Lit, 1-17.

Kaelble, H. (2004): Gibt es eine europäische Zivilgesellschaft?, in: Gosewinkel, D./ Rucht, D./ van Daele, W./ Kocka, J. (Hrsg.): Zivilgesellschaft - national und transnational. WZBJahrbuch 2003, Berlin: Edition Sigma, 267-284.

Kaufmann, F.-X. (2002): Sozialpolitik und Sozialstaat: Soziologische Analysen, Opladen: Leske und Budrich.

Luhmann, N. (1968): Vertrauen. Ein Mechanismus der Reduktion sozialer Komplexität, Stuttgart: Enke.

Luhmann, N. (1993): Wirtschaftsethik - als Ethik?, in: Wieland, J. (Hrsg.): Wirtschaftsethik und Theorie der Gesellschaft, Frankfurt a. M.: Suhrkamp, 134-147.

Pankoke, E. (2000): Subsidiäre Solidarität zwischen Markt und Staat. Der Dritte Sektor in den institutionellen Kulturen Europas, in: Universität Essen (Hrsg.): Essener Unikate, 14, 68-79.

Priller, E./ Zimmer, A. (2004): Dritte-Sektor-Organisationen zwischen „Markt“ und „Mission“, in: Gosewinkel, D./ Rucht, D./ van den Daele, W./ Kocka, J. (Hrsg.): Zivilgesellschaft - national und transnational. WBZ-Jahrbuch 2003, Berlin: Edition Sigma, 105-127.

Soosten, von J. (2001): Zivilgesellschaft, in: Honecker, M./ Dahlhaus, H./ Hübner, J. (Hrsg.): Evangelisches Soziallexikon, Stuttgart: Kohlhammer, 1846-1849.

Ulrich, P. (2000): Integrative Wirtschaftsethik: Grundlagenreflexion der ökonomischen Vernunft, in: Ethik und Sozialwissenschaften, Jg. 11, Heft 4, 553-567.

Ulrich, P. (2001): Integrative Wirtschaftsethik. Grundlagen einer lebensdienlichen Ökonomie, Bern u. a.: Haupt.

Zimmer, A. (2007): Vereine - Zivilgesellschaft konkret, 2. Aufl., Wiesbaden: VS-Verlag.

Zimmer, A./ Priller, E. (2005): Der Dritte Sektor im allgemeinen Diskurs, in: Birkhölzer, K./ Klein, A./ Priller, E./ Zimmer, A. (Hrsg.): Dritter Sektor/Drittes System. Theorie, Funktionswandel und zivilgesellschaftliche Perspektiven, Wiesbaden: VS-Verlag, 49-70. 